\title{
Impossibility of Distinguishing Two Preparations for a Pure State from No-signaling
}

\author{
Arun Kumar Pati \\ Harish-Chandra Research Institute, Jhunsi, Allahabad, India.E-mail:akpati@hri.res.in
}

Editors: Srikanth Radhakrishna \& Danko Georgiev

Article history: Submitted on September 2, 2020; Accepted on October 18, 2020; Published on October 22, 2020.

\section{A}

pure state of a physical system can be prepared in an infinite number of ways. Quantum theory dictates that given a pure state of a physical system it is impossible to distinguish two preparation procedures. Here, we show that the impossibility of distinguishing two preparation procedures for the same pure state follows from the no-signaling principle. Extending this result for a pure bipartite entangled state entails that the impossibility of distinguishing two preparation procedures for a mixed state follows from the impossibility of distinguishing two preparations for a pure bipartite state.

Quanta 2020; 9: 16-21.

\section{Introduction}

Quantum mechanics is one of the most successful theory that was developed in the last century. In early days of quantum mechanics, it was known in two different mathematical formulations, one is the matrix mechanics due to Heisenberg and the other is the wave mechanics due to Schrödinger. Later, it was shown that both these formulations are equivalent. Subsequently, von Neumann provided a natural framework using the axiomatic theory

(c) (1) This is an open access article distributed under the terms of the Creative Commons Attribution License CC-BY-3.0, which permits unrestricted use, distribution, and reproduction in any medium, provided the original author and source are credited. of Hilbert spaces and linear operators [1]. This mathematical formulation of quantum mechanics greatly influenced later developments. von Neumann's formulation of quantum mechanics in terms of vectors and operators in the abstract Hilbert space provided a general framework for the interpretation of quantum theory. Most notably, von Neumann's projection postulate is crucial when one tries to describe quantum measurement process in a physical system.

Unlike a classical system, a quantum system needs a different description of its state. In quantum mechanics, the state of a physical system, be it pure or mixed, is supposed to capture the complete description of a system [2]. If a system is described by a wavefunction, then we can denote it also by a pure state density operator. If a system is not prepared in a definite pure state, then we denote that by a mixed state density operator. The mixed state was introduced by von Neumann as the concept of statistical matrix for the description of an ensemble of systems which are not necessarily all in the same quantum state [3]. It is known that if we describe the system by a mixed state, then the same mixed state can be prepared in an infinite number of ways by probabilistic mixing of different pure states. Once the state is prepared, it is impossible in principle to distinguish two (or more) preparation procedures for a mixed state. In fact, if we can distinguish two preparations for a mixed state, then we could have signaling and we could violate the second law of thermodynamics [4]. 
Let us consider a system in a finite dimensional Hilbert space $\mathcal{H}^{N}$ which is separable, i.e., it admits a countable orthonormal basis. Every countable orthonormal basis set will represent eigenbasis of some Hermitian observables and correspondingly a possible preparation procedure (as explained in the sequel). If a physical system is in a pure state, that can also be prepared in an infinite number of ways. Since a Hilbert space $\mathcal{H}$ can have an infinite number of orthonormal basis sets, we can expand a pure state using any orthonormal basis. Consider two observables $A$ and $B$ with eigenbasis sets $\left\{\left|a_{n}\right\rangle\right\}$ and $\left\{\left|b_{n}\right\rangle\right\}$, respectively. Then, we know that we can express the pure state using the eigenbasis of the observables $A$, i.e., $|\psi\rangle=\sum_{n=1}^{N} \alpha_{n}\left|a_{n}\right\rangle$ or we could also expand the pure state using the eigenbasis of the observables $B$, i.e., $|\psi\rangle=\sum_{m=1}^{N} \beta_{m}\left|b_{m}\right\rangle$. Each one of these possible expansions represents one possible preparation procedure. In quantum mechanics, we can prepare a pure state starting from a fiducial pure state either by a unitary transformation, or by a general quantum operation such as a completely positive trace preserving (CPTP) map. Since this has to be a physical operation, and any physical operation can be realized by a unitary transformation of the fiducial state along with an ancillary state, without loss of generality, we consider here only unitary operation as a physically realizable process to prepare a pure state. For example, we can prepare $|\psi\rangle \in \mathcal{H}^{N}$ by choosing the initial state of the system as one of the eigenstates of the observable $A$, i.e., $\left|a_{0}\right\rangle$ and then we apply a unitary $U$ to obtain $|\psi\rangle$. In this case, we have $|\psi\rangle=U\left|a_{0}\right\rangle=\sum_{n}\left|a_{n}\right\rangle\left\langle a_{n}|U| a_{0}\right\rangle=\sum_{n} \alpha_{n}\left|a_{n}\right\rangle$, where $\alpha_{n}=\left\langle a_{n}|U| a_{0}\right\rangle$. Similarly, the other preparation procedure can be realized by choosing the initial state of the system as one of the eigenstates of the observable $B$, i.e., $\left|b_{0}\right\rangle$ and then we apply a unitary $V$ to obtain $|\psi\rangle$. In this case, we have $|\psi\rangle=V\left|b_{0}\right\rangle=\sum_{m}\left|b_{m}\right\rangle\left\langle b_{m}|V| b_{0}\right\rangle=\sum_{m} \beta_{m}\left|b_{m}\right\rangle$, where $\beta_{m}=\left\langle b_{m}|V| b_{0}\right\rangle$. However, once $|\psi\rangle$ is given to us, we cannot determine how this state has been prepared. What we ask in the next section is can this be formalized and this impossibility result obtained from no-signaling condition which is another fundamental tenet of any physical theory.

\section{Distinguishing two preparations}

In this section, we formalize the question what it means to distinguish two preparation procedures for a pure state. To convey the main result, we consider a quantum system in a two-dimensional Hilbert space, i.e., a qubit in a state $|\psi\rangle=\alpha|0\rangle+\beta|1\rangle \in \mathcal{H}^{2}$. This can be prepared starting from an initial state $|0\rangle$ (say along up-z-axis of a spin-half particle) and by applying a unitary $U(\alpha, \beta)$, i.e., $|\psi\rangle=U(\alpha, \beta)|0\rangle$. The same state can also be prepared starting from an initial state $|+\rangle$ (say up- $x$-axis of a spin-half particle) and by applying a different unitary $V(\alpha, \beta)$, i.e., $|\psi\rangle=V(\alpha, \beta)|+\rangle$. Now, quantum mechanics demands that if a pure state is a complete description of a physical system, then there is no way to distinguish different preparations. Otherwise, one may argue that the description is not complete. The question we address is whether the impossibility of distinguishing two preparation procedures for a pure state can be derived from some other known principle. To our surprise, we found that the impossibility of distinguishing two preparation procedures for a pure state has never been discussed in the literature, not to mention about how that follows from the no-signaling. Here, we show that the impossibility of distinguishing two (or more) preparations for a pure state actually follows from the no-signaling. We prove this by contradiction, i.e., we prove that if we can distinguish two different preparations for a pure state, then one can have signaling. But we know that we have to respect the no-signaling and hence it must be impossible to distinguish two different preparations for a pure state. Also, we argue that this impossibility result has a different status compared to other no-go theorems such as the impossibility of measuring without disturbance [5], the no-cloning in quantum information [6,7] and the no-deleting theorem [8]. Towards the end, we prove that the impossibility of distinguishing two preparation procedures for a mixed state follows from the impossibility of distinguishing two preparations for a pure bipartite entangled state.

Suppose that there is a machine which can distinguish two preparation procedures for a pure state. If the machine respects quantum mechanics, then that has to be represented by a physical operation. We can always realize a physical operation as a unitary evolution on a larger Hilbert space $\mathcal{H}^{2} \otimes \mathcal{H}^{2}$. Imagine that the physical operation is a unitary operation on the system and the machine state. Now, assume that the machine somehow knows the preparation procedure and the final state of the machine changes according to the preparation procedure. Thus, the unitary transformation that may distinguish two preparations for a qubit state is given by

$$
\begin{array}{ll}
U|0\rangle \otimes|A\rangle & \rightarrow\left|\psi_{0}\right\rangle \otimes\left|A_{U_{0}}\right\rangle, \\
V|+\rangle \otimes|A\rangle & \rightarrow\left|\psi_{+}\right\rangle \otimes\left|A_{V_{+}}\right\rangle,
\end{array}
$$

where $|A\rangle$ is the initial state of the machine, $\left|A_{U_{0}}\right\rangle$ is the final state of the machine if the pure state is prepared via $U|0\rangle$ and $\left|A_{V_{+}}\right\rangle$is the final state of the machine if the pure state is prepared via $V|+\rangle$. The states $\left|\psi_{0}\right\rangle$ and $\left|\psi_{+}\right\rangle$denote the fact that the pure state has been prepared starting from two different initial states $|0\rangle$ and $|+\rangle$, respectively. The 'preparation-distinguishing' machine, if it exists, then 
will change the final state of the machine according to the preparation procedure. Note that we need the states $\left|A_{U_{0}}\right\rangle$ and $\left|A_{V_{+}}\right\rangle$to be different, so that we can obtain information about the preparation procedures of the pure state. However, if this is to hold, then we have $1=$ $\left\langle\psi_{0} \mid \psi_{+}\right\rangle\left\langle A_{U_{0}} \mid A_{V_{+}}\right\rangle$. This implies that $\left|A_{U_{0}}\right\rangle$ and $\left|A_{V_{+}}\right\rangle$can never be different and hence there is no way to distinguish two preparation procedures for a pure state.

Note that the machine that we have defined above is distinct one compared to the machine that is supposed to measure two non-orthogonal quantum states without disturbance. In this case, the transformation is defined as

$$
\begin{array}{ll}
|0\rangle \otimes|A\rangle & \rightarrow \quad|0\rangle \otimes\left|A_{0}\right\rangle \\
|+\rangle \otimes|A\rangle & \rightarrow \quad|+\rangle \otimes\left|A_{+}\right\rangle .
\end{array}
$$

That this process is also impossible follows from the unitarity, because we can never be able to satisfy $1=\left\langle A_{0} \mid A_{+}\right\rangle$. This is paraphrased by saying that 'it is impossible to distinguish two non-orthogonal states without disturbance'. However, note that these two machines are completely different. This is because there is no way that we can go from (1) to (2) as the unitaries $U$ and $V$ in general will not commute with the global operation that realizes the process given in (1). Therefore, the hypothetical machine that can distinguishing two preparation procedures for a pure state is fundamentally different than the machine that is supposed to distinguish two non-orthogonal quantum states without disturbance. Therefore, this impossibility result is independent of the earlier one. We can also argue that it is independent of the no-cloning theorem [6,7]. First, note that if we know the complete preparation procedure, then we know the state of a qubit. But the converse is not true, i.e., knowing the state of a qubit is not same as knowing the preparation procedure. If we know the state of a qubit, then we can clone it, whereas here, even if we know the state we cannot distinguishing two preparation procedures. This shows that the present no-go theorem is different from the other no-go theorems such as the no-cloning [6,7, 9] and the no-deleting theorems [8].

\section{Distinguishing two preparations and signaling}

Here, we will show that distinguishing two different preparations for a pure state can actually lead to signaling. Imagine that Alice and Bob share an entangled EinsteinPodolsky-Rosen (EPR) pair [10,11] as given by

$$
\left|\Psi^{-}\right\rangle=\frac{1}{\sqrt{2}}(|0\rangle|1\rangle-|1\rangle|0\rangle) .
$$

The EPR state satisfies the property

$$
\begin{aligned}
\left|\Psi^{-}\right\rangle & =U(\alpha, \beta) \otimes U(\alpha, \beta)\left|\Psi^{-}\right\rangle \\
& =\frac{1}{\sqrt{2}}(|\psi\rangle|\bar{\psi}\rangle-|\bar{\psi}\rangle|\psi\rangle),
\end{aligned}
$$

where

$$
\begin{aligned}
& |\psi\rangle=\alpha|0\rangle+\beta|1\rangle=U(\alpha, \beta)|0\rangle, \\
& |\bar{\psi}\rangle=\alpha^{*}|1\rangle-\beta^{*}|0\rangle=U(\alpha, \beta)|1\rangle .
\end{aligned}
$$

This invariance property of singlet is equivalent to

$$
\begin{aligned}
U^{\dagger}(\alpha, \beta) \otimes I\left|\Psi^{-}\right\rangle & =I \otimes U(\alpha, \beta)\left|\Psi^{-}\right\rangle \\
& =\frac{1}{\sqrt{2}}(|0\rangle U|1\rangle-|1\rangle U|0\rangle) .
\end{aligned}
$$

Physically, this means that if Alice applies $U^{\dagger}(\alpha, \beta)$ on her particle this is equivalent to applying $U(\alpha, \beta)$ on Bob's particle. Similarly, the invariance property for the singlet implies that we have

$$
\begin{aligned}
V^{\dagger}(\alpha, \beta) \otimes I\left|\Psi^{-}\right\rangle & =I \otimes V(\alpha, \beta)\left|\Psi^{-}\right\rangle \\
& =\frac{1}{\sqrt{2}}(|+\rangle V|-\rangle-|-\rangle V|+\rangle),
\end{aligned}
$$

with the notion that $|\psi\rangle=V(\alpha, \beta)|+\rangle$ and $|\bar{\psi}\rangle=V(\alpha, \beta)|-\rangle$. Now, let us encode one classical bit in Alice's action, i.e., if she receives 0 , then she applies $U^{\dagger}(\alpha, \beta)$ on her particle and if she receives 1 , then she applies $V^{\dagger}(\alpha, \beta)$ on her particle. These two choices by Alice allow us to have the possibility of two different preparations at Bob's end. Now assume that Bob has a hypothetical machine which can distinguish two preparation procedures for a pure state. Bob attaches the machine and allows the transformation as given by

$$
\begin{array}{ll}
U|0\rangle|A\rangle \rightarrow\left|\psi_{0}\right\rangle\left|A_{U_{0}}\right\rangle, & V|+\rangle|A\rangle \rightarrow\left|\psi_{+}\right\rangle\left|A_{V_{+}}\right\rangle, \\
U|1\rangle|A\rangle \rightarrow\left|\bar{\psi}_{1}\right\rangle\left|A_{U_{1}}\right\rangle, & V|-\rangle|A\rangle \rightarrow\left|\bar{\psi}_{-}\right\rangle\left|A_{V_{-}}\right\rangle .
\end{array}
$$

Then, depending on the two choices of preparations of a pure state, we have

$$
\begin{aligned}
& \frac{1}{\sqrt{2}}(|0\rangle U|1\rangle|A\rangle-|1\rangle U|0\rangle|A\rangle) \rightarrow \\
& \frac{1}{\sqrt{2}}\left(|0\rangle\left|\bar{\psi}_{1}\right\rangle\left|A_{U_{1}}\right\rangle-|1\rangle\left|\psi_{0}\right\rangle\left|A_{U_{0}}\right\rangle\right), \\
& \frac{1}{\sqrt{2}}(|+\rangle V|-\rangle|A\rangle-|-\rangle V|+\rangle|A\rangle) \rightarrow \\
& \frac{1}{\sqrt{2}}\left(|0\rangle\left|\bar{\psi}_{-}\right\rangle\left|A_{V_{-}}\right\rangle-|1\rangle\left|\psi_{+}\right\rangle\left|A_{V_{+}}\right\rangle\right) .
\end{aligned}
$$

Now, the two preparation procedures give two different density matrices at Bob's end. These are given by

$$
\begin{gathered}
\rho_{B}^{0}=\frac{1}{2}\left[\left|\bar{\psi}_{1}\right\rangle\left\langle\bar{\psi}_{1}|\otimes| A_{U_{1}}\right\rangle\left\langle A_{U_{1}}|+| \psi_{0}\right\rangle\left\langle\psi_{0}|\otimes| A_{U_{0}}\right\rangle\left\langle A_{U_{0}}\right|\right], \\
\rho_{B}^{+}=\frac{1}{2}\left[\left|\bar{\psi}_{-}\right\rangle\left\langle\bar{\psi}_{-}|\otimes| A_{V_{-}}\right\rangle\left\langle A_{V_{-}}|+| \psi_{+}\right\rangle\left\langle\psi_{+}|\otimes| A_{V_{+}}\right\rangle\left\langle A_{V_{+}}\right|\right],
\end{gathered}
$$


where $\rho_{B}^{0}$ is the result of one preparation procedure and $\rho_{B}^{+}$is the result of the other preparation procedure. Since these two density matrices are different, Bob can infer Alice's action, thus revealing one bit of information without any communication from Alice. This would lead to signaling. Therefore, from the no-signaling, we can argue that it is impossible to distinguish two preparation procedures for a pure state. This is in the same spirit as proving the no-cloning and the no-deleting from the no-signaling principle [7, 12, 13].

This means that any device that can distinguish two or more preparation procedures for a pure quantum state has to be outside the realm of quantum theory. Thus, the fundamental description of a physical system by a pure state is intimately connected to the no-signaling condition. For another perspective on distinguishing and signaling, interested readers can see the recent work by Srivastava et al. [14]. Both proofs, the one by us and the one given by Srivastava et al. [14] were obtained in parallel.

\section{Impossibility of distinguishing two preparations for a mixed state}

In quantum mechanics, a density matrix can have infinite number of decompositions (proper mixtures) and it is impossible to distinguish two preparation procedures. Also, we know that a mixture (improper) occurs when we trace out one of the subsystem of an entangled state. First, note that a pure bipartite entangled state can also be prepared in an infinite number of ways and quantum theory dictates that it is impossible to distinguish two or more preparations for a pure entangled state. Now, suppose, we ask the question: which one is more fundamental in nature, i.e., (i) impossibility of distinguishing two preparations for a pure bipartite entangled state or (ii) impossibility of distinguishing two preparations for a mixed state? To be more specific, does (ii) follow from (i) or vice versa? In what follows, we show that the impossibility to distinguish two preparations for a pure bipartite entangled state indeed implies the impossibility of distinguishing two preparation procedures for a mixed state.

Consider two preparation procedures of a pure bipartite state $|\Psi\rangle_{A B} \in \mathcal{H}^{N} \otimes \mathcal{H}^{N}$ which are expressed as $\sum_{n m} C_{n m}\left|\psi_{n}\right\rangle \otimes\left|\phi_{m}\right\rangle$ and $\sum_{\mu \nu} \alpha_{\mu \nu}\left|a_{\mu}\right\rangle \otimes\left|b_{\nu}\right\rangle$. Extending our earlier result to a pure bipartite state, we can show that it is also impossible to distinguish two different preparations. Now, these two preparation procedures for a pure state will result in two possible preparations for the density matrix of either subsystem [15]. For example, if we trace out the second subsystem, we will have density matrix $\rho_{A}=\sum_{m}\left|\tilde{\psi}_{m}\right\rangle\left\langle\tilde{\psi}_{m}\right|$, where $\left|\tilde{\psi}_{m}\right\rangle=\sum_{n} C_{n m}\left|\psi_{n}\right\rangle$ are unnor- malized and non-orthogonal states with $\sum_{m}\left\|\tilde{\psi}_{m}\right\|^{2}=1$. Similarly, for the other preparation procedure, if we trace out the second subsystem, then the density matrix $\rho_{A}=\sum_{v}\left|\tilde{a}_{v}\right\rangle\left\langle\tilde{a}_{v}\right|$, where $\left|\tilde{a}_{v}\right\rangle=\sum_{\mu} \alpha_{\mu \nu}\left|a_{\mu}\right\rangle$ are unnormalized and non-orthogonal states with $\sum_{v}\left\|\tilde{a}_{v}\right\|^{2}=1$.

Now, suppose that there is a physical operation that can perfectly distinguish two preparations for the same mixed state. This means that the 'preparation-distinguishing' machine can result in two different states of the system and the machine as $\rho_{A E}^{(1)}$ and $\rho_{A E}^{(2)}$ corresponding to two different preparation procedures, respectively, with $D\left(\rho_{A E}^{(1)}, \rho_{A E}^{(2)}\right)=1$. Here $D$ is a measure of distinguishing two different preparations which are labeled as ' 1 ' and ' 2 '. This physical operation can also be realized on a purified Hilbert space that results in two possible states $\left|\Psi_{A B E}^{(1)}\right\rangle$ and $\left|\Psi_{A B E}^{(2)}\right\rangle$ according to two different preparation procedures. Note that the distinguishabilty measure decreases under partial tracing [16]. Therefore, we have

$$
D\left(\rho_{A E}^{(1)}, \rho_{A E}^{(2)}\right) \leq D\left(\Psi_{A B E}^{(1)}, \Psi_{A B E}^{(2)}\right) .
$$

Since we have already assumed that $D\left(\rho_{A E}^{(1)}, \rho_{A E}^{(2)}\right)=1$, this then implies that $D\left(\Psi_{A B E}^{(1)}, \Psi_{A B E}^{(2)}\right) \geq 1$. If the distinguishable measure satisfies $0 \leq D \leq 1$, then it must be true that $D\left(\Psi_{A B E}^{(1)}, \Psi_{A B E}^{(2)}\right)=1$, i.e., we can distinguish two preparations for a pure bipartite entangled state perfectly. But we know that we cannot distinguish two different preparations for the same pure entangled state and hence it is impossible to distinguish two preparations for the same mixed state.

It may be noted that we cannot prove the opposite, i.e., we cannot prove that the impossibility of distinguishing two preparations for a pure bipartite entangled state follows from the impossibility of distinguishing two preparations for a mixed state. Thus, our result is a stronger than impossibility of distinguishing two preparations for a mixed state-which is central to quantum information.

\section{Conclusion}

In the context of mixed states, one can have manifestly distinct ensemble realizations of the same mixed state. Thus, it is a non-trivial observation that different preparations of the same mixed state cannot be distinguished. By contrast, in the context of a pure state, although there can be infinitely many representations in different bases, still since a pure state can have only one ensemble realization, it may appear that the idea of indistinguishability of different preparations of the same pure state is not on the same footing. Here, we argue that this is not the case. Because, even in the case of pure ensemble, we can imagine that we divide the ensemble to two sub-ensembles, where one sub-ensemble has been prepared using one procedure 
and the other sub-ensemble has been prepared using a different preparation procedure. Then, the question is can we retrace back and 'somehow' know how those two pure sub-ensembles have been prepared. One may say that if two preparations could be distinguished, we would simply say that they correspond to distinct pure states, rather than recognize it as a case of distinguishing two preparations. Again, one should remember that the question we address is two different preparations are not giving rise to two distinct pure states, rather they result in the same pure states starting from two different pasts. Here, we ask can the no-signaling has some implication on the fact that a pure state is an equivalence class of different preparations that behave identically under all possible measurements. Then, we have shown that if there is a hypothetical device that can distinguish two or more preparations for the same pure state then that can lead to signaling.

In quantum theory, the preparation of a physical system and the measurement procedure play fundamental role. As von Neumann would emphasize, the measurement process though always entails an outcome which may be random, an experimentalist must be able to reproduce the preparation and measurement procedures. A reproducible preparation of a physical system is represented by a pure state (in the case of closed system) or by a density operator (in the case of open system). There are infinite number of ways in which a given pure state can be prepared and hence there is an infinite number of pasts associated to a present pure state of a physical system. Quantum theory tells us that once the state is prepared in a pure state, then there is no way to reveal its preparation procedure. The new impossibility theorem says that even if we know the present state it is impossible for us to know its past, namely, from where it has started and how it has reached the present state. We have strengthened this impossibility result and have shown that the impossibility of distinguishing two different preparations for a pure state follows from the no-signaling principle. Remarkably, we have also proved that the impossibility of distinguishing two preparations for the same mixed state follows from a more fundamental result that it is impossible to distinguish two different preparations for the same pure bipartite entangled state. This is another non-trivial implication of our result. This impossibility result has a different status compared to other no-go theorems such as the no-cloning and the no-deleting theorems in quantum information. The latter two no-go theorems do not hold if we know the state. However, the new impossibility result is truly quantum in origin without any classical counterpart.

\section{Acknowledgment}

I thank Ujjwal Sen and Chirag Srivastava for useful discussions.

\section{References}

[1] J. von Neumann. Mathematische Begründung der Quantenmechanik. Nachrichten von der Gesellschaft der Wissenschaften zu Göttingen, Mathematisch-Physikalische Klasse 1927; pp. 1-57. https://eudml.org/doc/59215.

[2] P. A. M. Dirac. The Principles of Quantum Mechanics. 4th Edition. Oxford University Press, Oxford, 1967.

[3] J. von Neumann. Mathematische Grundlagen der Quantenmechanik. Springer, Berlin, 1932. doi: 10.1007/978-3-642-61409-5.

[4] A. Peres. Quantum Theory: Concepts and Methods. Vol. 72 of Fundamental Theories of Physics. Kluwer, New York, 1993. doi:10.1007/0306-47120-5.

[5] J. L. Park. The concept of transition in quantum mechanics. Foundations of Physics 1970; 1(1):2333. doi:10.1007/bf00708652.

[6] W. K. Wootters, W. H. Zurek. A single quantum cannot be cloned. Nature 1982; 299(5886):802-803. doi:10.1038/299802a0.

[7] D. Dieks. Communication by EPR devices. Physics Letters A 1982; 92(6):271-272. doi:10.1016/ 0375-9601(82) 90084-6.

[8] A. K. Pati, S. L. Braunstein. Impossibility of deleting an unknown quantum state. Nature 2000; 404(6774):164-165. arXiv:quant-ph/ 9911090. doi:10.1038/404130b0.

[9] H. P. Yuen. Amplification of quantum states and noiseless photon amplifiers. Physics Letters A 1986; 113(8):405-407. doi:10.1016/0375$9601(86) 90660-2$.

[10] A. Einstein, B. Podolsky, N. Rosen. Can quantummechanical description of physical reality be considered complete?. Physical Review 1935; 47(10):777780. doi:10.1103/PhysRev.47.777

[11] D. Bohm, Y. Aharonov. Discussion of experimental proof for the paradox of Einstein, Rosen, and Podolsky. Physical Review 1957; 108(4):1070-1076. doi:10.1103/PhysRev.108.1070. 
[12] N. Gisin. Quantum cloning without signaling. [15] L. P. Hughston, R. Jozsa, W. K. Wootters. A Physics Letters A 1998; 242(1):1-3. doi:10. 1016/S0375-9601(98)00170-4

[13] A. K. Pati, S. L. Braunstein. Quantum deleting and signalling. Physics Letters A 2003; 315(3):208-212. doi:10.1016/s0375-9601(03)01047-8.

[14] C. Srivastava, S. Das, A. SenDe, U. Sen. Signaling versus distinguishing different preparations of same pure quantum state. Journal of Physics A: Mathematical and Theoretical 2020; 53(27):275302. complete classification of quantum ensembles having a given density matrix. Physics Letters A 1993; 183(1):14-18. doi:10.1016/03759601(93) 90880-9.

[16] M. A. Nielsen, I. L. Chuang. Quantum Computation and Quantum Information. Cambridge University Press, Cambridge, 2010. doi:10.1017/ cbo9780511976667. 